WellBeing International

WBI Studies Repository

8-2008

\title{
A Note on the Effectiveness of Behavioural Rehabilitation for Reducing Inter-Dog Aggression in Shelter Dogs
}

\author{
Jane S. Orihel \\ University of British Columbia \\ David Fraser \\ University of British Columbia
}

Follow this and additional works at: https://www.wellbeingintlstudiesrepository.org/intbeh

Part of the Animal Studies Commons, Behavior and Ethology Commons, and the Comparative Psychology Commons

\section{Recommended Citation}

Orihel, J. S., \& Fraser, D. (2008). A note on the effectiveness of behavioural rehabilitation for reducing inter-dog aggression in shelter dogs. Applied Animal Behaviour Science, 112(3), 400-405.

This material is brought to you for free and open access by WellBeing International. It has been accepted for inclusion by an authorized administrator of the WBI Studies Repository. For more information, please contact wbisr-info@wellbeingintl.org.

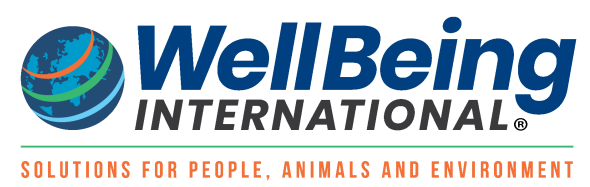




\title{
A Note on the Effectiveness of Behavioural Rehabilitation for Reducing Inter-Dog Aggression in Shelter Dogs
}

Jane S. Orihel and David Fraser

University of British Columbia

\begin{abstract}
KEYWORDS
canine, dog behaviour, rehabilitation, inter-dog aggression, shelter dogs

ABSTRACT

The effectiveness of a rehabilitation program for reducing inter-dog aggression was evaluated at the municipal animal shelter. Sixteen dogs (of 60 examined) met the study criteria of medium inter-dog aggression as determined by an inter-dog aggression test. These dogs received a 10-day treatment of daily rehabilitation for 30 min (rehabilitation group, $n=9$ ) or daily release into an outdoor enclosure for 30 min (control group, $n=7$ ). Rehabilitation consisted of desensitising and counter-conditioning dogs to the approach of other "stimulus" dogs. Most dogs in the rehabilitation group showed a decline in aggression scores when re-tested after the last treatment (day 11), and differed significantly from the control dogs which showed either an increase or no change in aggression scores $(U=8.5, P<0.01)$. Rehabilitation dogs also showed lower frequencies of aggressive body postures ("facing the stimulus dog", $P<0.05$, and "stiff posture", $P<0.10$ ) and higher frequencies of less assertive postures ("ears back", $P<$ 0.05 , and "lowered neck", $P<0.10$ ) on day 11 . The differences between groups were no longer significant when a reduced sample of dogs was tested 1 week after rehabilitation ended (day 18). The study shows short-term reduction of inter-dog aggression through rehabilitation, but further work is needed on effective ways of maintaining the behavioural change.
\end{abstract}

\section{Introduction}

Inter-dog aggression is a common behavioural complaint of dog owners (Voith, 1980). Aggression towards other animals has been reported to be a common reason for dogs to relinquished and/or returned to an animal shelter for behavioural reasons (Wells and Hepper, 2000). Aggression is also a serious management and legal concern for shelters, as workers may become injured when separating fighting dogs, and shelters may face legal action should an adopted dog prove aggressive. 
The shelter environment may also contribute to these problems. Housing dogs in social and spatial restriction have been associated with physiological changes (Hennessy et al., 1997), as well as behavioural disturbances such as stereotypes, barking, and aggression (Mertens and Unshelm, 1996). To prevent prolonged confinement of problem dogs, many shelters attempt to rehabilitate dogs with modifiable aggression problems (Orihel et al., 2005). Treatment commonly includes behaviour modification (Voith, 1980; Overall, 1997a). Such treatment has been moderately successful in clinical practice with owned dogs (Sherman et al., 1996), but remains unproven for shelter dogs. Furthermore, rehabilitation methods used by different shelters are diverse and shelter staffs have expressed the mixed levels of confidence in their respective programs (Orihel et al., 2005). Accordingly, this study compared shelter dogs that received rehabilitation for inter-dog aggression and control dogs that did not.

\section{Methods}

\subsection{Selection of dogs}

Sixty dogs admitted to a municipal animal shelter were screened for inter-dog aggression after an adaptation period of 4-10 days at the shelter. To reduce variation, puppies, seniors and dogs $<15 \mathrm{~kg}$ (as estimated by shelter staff) were excluded, as were dogs that showed aggression to staff.

Five inter-dog aggression tests (adapted from Netto and Planta, 1997) exposed the dogs to other "stimulus" dogs. The five tests were done in the same order and required 1-2 min each with 2-5 min interval between tests. Two female researchers alternated between the role of observer and stimulus dog handler. Observations were recorded on checklists. The tests were as follows: (1) a "friendly" (defined below) stimulus dog of either gender was passed by the test dog's kennel; (2) the test dog was walked past other kennelled dogs; (3) a "friendly" stimulus dog of the same gender was walked past the dog at a distance of $1.2 \mathrm{~m}$; (4) a "confident" stimulus dog of the same gender was walked towards the dog starting at $15 \mathrm{~m}$, and stopping when the test dog became aggressive or, otherwise, when the stimulus dog was $1.2 \mathrm{~m}$ from the test dog; (5) test 4 was repeated with a second "confident" stimulus dog of the same gender.

Dogs' responses in each test were rated on two behavioural traits: aggression and fearfulness. Five-point scales for each trait were used to rate the intensity of the behaviours, where $1=$ no visible signs of the behaviour and $5=$ extreme signs of the behaviour (adapted from Overall, 1997a). Scores for each trait were combined over the five tests for each dog; this resulted in a "total score" for each of aggression and fearfulness, ranging from 5 to 25. Additionally, the presence (1) or absence (0) of 62 behaviours and postures associated with agonistic behaviour in dogs (Overall, 1997b; van den Berg et al., 2003) was recorded for each test. For each dog, a total score of 0-5 was calculated for each behaviour and posture according to the number of tests in which the item was seen.

Based on a pilot study, dogs were judged suitable for the experiment if they had a total aggression score of 10-18, a total fearfulness score of $5-15$, and an aggression score $\geq 3$ (moderate to extreme) in one of tests 3,4 or 5 (outdoor tests simulating encounters in everyday life). Two dogs were excluded because they displayed three or more of five behaviours (muzzle licking, rapid panting, yawning, raising forepaw and blowing out lips) believed to indicate anxiety or stress in dogs (Voith and Borchelt, 1996).

Stimulus dogs were shelter residents that had displayed little or no inter-dog aggression during testing. Dogs were classified as "friendly" if they showed almost no sign of aggression (total score $<8$ ) and little sign of fearfulness (total score $<10$ ). Dogs were classified as "confident" if they showed not more than modest aggression (total score $<10$ ) and no sign of fearfulness (total score $=5$ ). Dogs were not used if they displayed more than three behaviours associated with anxiety or stress (Voith and Borchelt, 1996). 


\subsection{Housing}

Dogs were housed individually in indoor concrete kennels $(2.0 \mathrm{~m} \times 1.3 \mathrm{~m})$. Dogs were fed at least $1 \mathrm{~h}$ before testing or treatment. On the testing days and 10 treatment days, dogs received a 5 min release into an outdoor enclosure between 8:00 and 9:00 h, a 30 min treatment between 11:30 and 14:30 h, a 15 min walk between $14: 30$ and $17: 30 \mathrm{~h}$, and a 10 min release into an enclosure between 18:00 and 21:00 $\mathrm{h}$.

\subsection{Experimental treatments}

Sixteen of the 60 dogs that underwent screening met the criteria for participants. One day after testing, these dogs were assigned to one of two 10-day treatments. Nine dogs (rehabilitation group) received daily rehabilitation for inter-dog aggression while seven dogs (control group) received daily release into an outdoor enclosure. Dogs were allocated to groups using restricted randomisation based on their pretreatment (day 0) aggression scores and gender. Rehabilitation dogs were four males (all neutered) and five females (two intact, three neutered) and control dogs were four males (three intact, one neutered) and three females (two intact, one neutered).

Rehabilitation was performed in a $36.5 \mathrm{~m} \times 7.3 \mathrm{~m}$ outdoor enclosure marked with initial positions for the participant and stimulus dog (23 m apart), plus three intermediate positions, 13, 8 and $5.5 \mathrm{~m}$ apart. Participant dogs wore a body harness (Sense-ation ${ }^{\mathrm{TM}}$, Bedfordshire, England, UK) and a head-collar (Halti®, Bedfordshire, England, UK) attached to a double-ended leash. Rehabilitation group dogs received daily 30 min sessions of repeated exposure to the approaches of individual stimulus dogs at successively closer distances (desensitisation). Initially, dogs were exposed to friendly, neutered stimulus dogs, progressing to friendly, intact dogs, then confident, neutered dogs and finally, confident, intact dogs over the 10 days. To replace undesirable behaviour with appropriate behaviour (counter-conditioning), dogs were commanded to sit or make eye contact with the handler. Dogs were rewarded intermittently for relaxed behaviour (positive reinforcement) with verbal praise and food treats (Jerky Treats ${ }$, DLM Foods Canada Corp, Ontario, Canada). Aggressive signals (e.g. direct staring and growling) were interrupted by using the head-collar to direct the dog's head and body away from the stimulus dog. To standardise the training between dogs, the trainer and stimulus dog handler alternated roles daily for each dog.

Control dogs were released individually into a $20 \mathrm{~m} \times 25 \mathrm{~m}$ outdoor enclosure with water and toys for 30 min each day. A person was present during releases but had little or no interaction with the dog.

The inter-dog aggression test was repeated one day after the treatment was completed (day 11) using the same observer. Dogs were returned to the shelter's regular schedule after day 11 and became available for neutering and adoption by the public. To assess the effects of the treatment 1 week after the first posttreatment test, the 12 dogs remaining in the shelter on day 18 were re-tested.

A subset of 22 pre- and post-treatment tests chosen according to convenience were videotaped for between- and within-observer reliability. Each observer independently scored the tests on two occasions separated by 1 month. In addition, a "naïve" observer rated aggression for 11 videotaped post-treatment tests (also chosen according to convenience) of rehabilitation and control group dogs in case the expectations of the "informed" observers affected their scoring.

\subsection{Data analysis}

Difference scores for aggression were calculated for each dog by subtracting the dog's aggression score in each post-treatment test (days 11 and 18) from its score in the pre-treatment test (day 0). Nonparametric tests were used because the data did not appear to be normally distributed. Mann-Whitney $U$ tests (Siegel, 1956) were used to test whether rehabilitation and control groups differed in the difference 
scores for aggression. Mann-Whitney $U$-tests were also used to test for differences between the two groups in day 0 aggression scores. Wilcoxon signed-ranks tests (Siegel, 1956) were used to test whether aggression scores changed significantly between different test days for dogs within each treatment group.

Thirty-two of the 62 ethogram items occurred in $<5 \%$ of the 60 initial pre-treatment tests and were excluded. To avoid an excessive number of comparisons, a preliminary Wilcoxon signed-ranks test was used to identify nine ethogram items which showed a change $(P<0.10)$ from days 0 to 11 , or to day 18 in one or both groups. Difference scores were calculated by subtracting the frequency score for each ethogram item in each post-treatment test (days 11 and 18) from its score in the pre-treatment test (day $0)$. Mann-Whitney $U$-tests were used to test whether difference scores for the nine items differed significantly between groups.

To test within-observer reliability for each observer, Spearman correlation coefficients were calculated between the scale ratings from the first and second scorings. For the between-observer reliability, Spearman correlation coefficients were calculated between the ratings of the two observers for aggression and fearfulness. To test for effects of the observers' expectations, total aggression scores for rehabilitation and control dogs produced by the "naïve" observer were compared to those of the "informed" observers.

Statistical tests were one-tailed where we formulated explicit hypotheses, specifically, that rehabilitation dogs would have lower aggression scores after treatment than before, and lower difference scores for aggression than control dogs. Other tests were two-tailed. Differences at $P<0.10$ and beyond are reported.

\section{Results}

Dogs in the rehabilitation and control groups showed similar pre-treatment (day 0) test scores for aggression (median $=12$ versus 11, respectively; $U=27.5, P=0.67$ ). Six of nine dogs in the rehabilitation group showed a decline in aggression score from days 0 to the first test after treatment (day 11). The median difference score for the nine dogs was $-2(W=27.5, P=0.09)$. In contrast, five of seven control dogs increased their aggression scores from day 0 to 11 , giving an overall median difference score of $+2(W=15, P<0.05)$. The difference scores differed significantly between the rehabilitation and control groups $(U=8.5, P<0.01)$. When dogs were re-tested 1 week after treatment was completed (day 18), aggression scores for the rehabilitation dogs had largely returned to their initial values on day 0 ( median $=14)$.

The rehabilitation group showed a reduction from days 0 to 11 in the frequency of "facing the stimulus dog" $(P<0.05)$ and in "stiff posture" $(P<0.10)$. Rehabilitation dogs also showed an increase in "ears back" $(P<0.05)$ and "lowered neck" $(P<0.10)$. The control group showed an increase from day 0 to 11 in growling $(P<0.10)$ and muzzle licking $(P<0.10)$. The difference scores differed between the two groups for "ears back" $(U=7.5, P<0.05)$.

On day 18 , as on day 11 , rehabilitation dogs showed a decline from day 0 in the frequency of "facing the stimulus dog" $(P<0.10)$, and an increase in "lowered neck" $(P<0.10)$ and "ears back" $(P<0.10)$. Control dogs showed an increase in growling $(P<0.05)$ and "ears up" $(P<0.10)$ on day 18. The difference scores for "ears up" differed significantly between the groups $(U=2.5, P=0.01)$.

The mean Spearman correlation for the within-observer ratings was 0.90 for aggression and 0.62 for fearfulness. Between-observer reliabilities were 0.82 for aggression and 0.56 for fearfulness. The naïve observer and the informed observers were in exact agreement over the difference in aggression scores 
between the rehabilitation and control groups: for both categories of observers, the median score for rehabilitation dogs was lower than for control dogs by a value of 2.5 .

\section{Discussion}

This study provides evidence of short-term reduction of inter-dog aggression through a rehabilitation program. Most rehabilitation dogs showed a decrease from pre-treatment (day 0 ) to post-treatment (day 11) in aggression scores, lowered frequencies of body postures that are associated with challenge or aggression ("facing the stimulus dog" and "stiff posture"), and increased frequencies of "lowered neck" and "ears back", postures which commonly represent a dog that is less "actively assertive" (Overall, 1997b). The large number of comparisons left scope for low $P$ values to occur by chance, but all the trends observed were consistent with rehabilitation reducing aggressiveness.

The different components of rehabilitation may have influenced aggression in different ways. Desensitisation and positive reinforcement can reduce anxiety by weakening the association between the threatening stimulus and negative emotional reactions. Counter-conditioning can allow dogs to better control and cope with the aggression-provoking situation by providing alternative behavioural options (Lindsay, 2000). Rehabilitation also involved positive human interaction, which has been shown to reduce the behavioural and physiological stress responses of shelter dogs (Hennessy et al., 1998). Further work is needed to identify how these different components of rehabilitation contributed to the temporary reduction in aggression.

Dogs that did not receive rehabilitation showed a significant increase in aggression scores on day 11 compared with their scores on day 0 . Control dogs also showed more occurrences of growling and higher ear posture ("ears up"); these behaviours are often seen in animals establishing dominance or behaving aggressively (Overall, 1997b). In addition, control dogs showed a greater increase in muzzle licking (thought to denote anxiety) than rehabilitation dogs. However, it should be acknowledged that more neutered males and spayed females participated in the rehabilitation than in the control group. Because hormonal activity influences inter-dog aggression (Sherman et al., 1996), the group differences in reproductive status in our study may have influenced the dogs' responsiveness to the treatment.

When the remaining dogs were re-tested 1 week after the last treatment (day 18), there was no evidence of a sustained effect of rehabilitation on aggression. This suggests that 10 days of rehabilitation were not sufficient to prevent later increases in aggression once treatment ended. However, the evidence is relatively weak because of the reduced sample size on day 18. Additionally, this finding pertains to dogs that stayed in the shelter environment after rehabilitation ended. Nonetheless, rehabilitation dogs showed behavioural changes from day 0 to 18, for example fewer occurrences of "facing the stimulus dog", and more occurrences of "lowered neck" and "ears back", that suggest a reduction in assertive behaviour. In contrast, most control dogs showed an increase in aggression on day 18, and more occurrences of "ears up" and growling.

\section{Conclusions}

Since the beneficial effects of rehabilitation on inter-dog aggression declined rapidly, continued treatment is likely necessary for maintaining the behavioural change. Long-term studies are warranted to determine the optimal frequency and duration of rehabilitation. By introducing validated rehabilitation programs, shelters could potentially improve the welfare of aggressive dogs while at the shelter and increase their chances of successful adoption. Educating adopters on how to manage inter-dog aggression may further support the retention of the improved behaviours in the new home. 


\section{Acknowledgements}

We gratefully acknowledge the staff and volunteers at the participating shelters, Zuzana Zemanova, Darcie van Peteghem, and Elsie Dawn Parsons for their assistance in conducting the study, and Dr. Rebecca Ledger for input to the research design. The project was funded by the Natural Sciences and Engineering Research Council of Canada through the Industrial Research Chair in Animal Welfare with contributions from our sponsors listed at http://www.landfood.ubc.ca/animalwelfare/.

\section{References}

Hennessy, M.B., Davis, H.N.,Williams, M.T., Mellott, C., Douglas, C.W., 1997. Plasma cortisol levels of dogs at a county animal shelter. Physiol. Behav. 62, 485-490.

Hennessy, M.B.,Williams, M.T., Miller, D.D., Douglas, C.W., Voith, V.L., 1998. Influence of male and female petters on plasma cortisol and behaviour: can human interaction reduce the stress of dogs in a public animal shelter? Appl. Anim. Behav. Sci. 61, 63-77.

Lindsay, S.R., 2000. Applied Dog Behavior and Training, vol. 1. lowa State University Press, Philadelphia, PA. Mertens, P.A., Unshelm, J., 1996. Effects of group and individual housing on the behavior of kenneled dogs in animal shelters. Anthrozoös 9, 40-51.

Netto, W.J., Planta, D.J.U., 1997. Behavioural testing for aggression in the domestic dog. Appl. Anim. Behav. Sci. 52, 243-263.

Orihel, J.S., Ledger, R.A., Fraser, D., 2005. A survey of the management of inter-dog aggression by animal shelters in Canada. Anthrozoös 18, 273-287.

Overall, K.A., 1997a. Canine aggression. Clinical Behavioral Medicine for Small Animals. Mosby, St. Louis, MS, pp. 88-137.

Overall, K.A., 1997b. Normal canine behavior. Clinical Behavioral Medicine for Small Animals. Mosby, St. Louis, MS, pp. 9-44.

Sherman, C.K., Reisner, I.R., Taliaferro, L.A., Houpt, K.A., 1996. Characteristics, treatment, and outcome of 99 cases of aggression between dogs. Appl. Anim. Behav. Sci. 47, 91-108.

Siegel, S., 1956. Nonparametric Statistics for the Behavioral Sciences. McGraw-Hill, Singapore, pp. 75127.

van den Berg, L., Schilder, M.B.H., Knol, B.W., 2003. Behavior genetics of canine aggression: behavioral phenotyping of golden retrievers by means of an aggression test. Behav. Genet. 33, 469-483.

Voith, V.L., 1980. Inter-male aggression in dogs. Mod. Vet. Pract. 61, 256-258.

Voith, V.L., Borchelt, P.L., 1996. Fears and phobias in companion animals: update. In: Voith, V.L., Borchelt, P.L. (Eds.), Readings in Companion Animal Behaviour. Veterinary Learning Systems, Trenton, NJ, pp. 140-152.

Wells, D.L., Hepper, P.G., 2000. Prevalence of behaviour problems reported by owners of dogs purchased from an animal rescue shelter. Appl. Anim. Behav. Sci. 69, 55-65. 